NASACONTRACTOR REPOR T

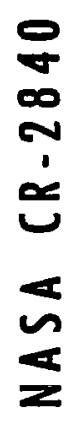

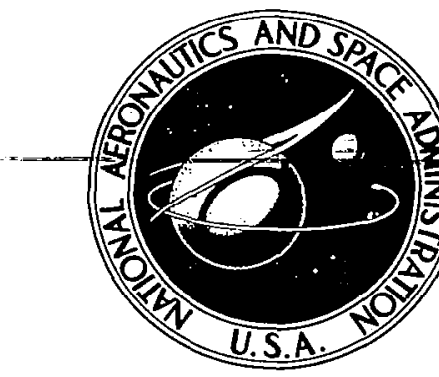

LOAN COPY: RETURN TO AFWL TECHNICAL LIERAFY KIRTLAND AFB, N. M.

\title{
ACOUSTIC EMISSION DURING UNLOADING OF ELASTICALLY STRESSED MAGNESIUM ALLOY
}

Samson S. Lee and James H. Williams, Jr.

Prepared by

MASSACHUSETTS INSTITUTE OF TECHNOLOGY

Cambridge, Mass. 02139

for Lewis Research Center

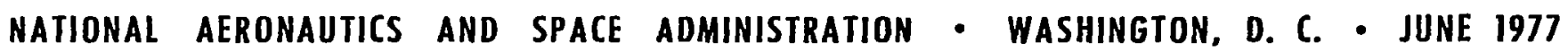


0061690



* For sale by the National Technical Information Service. Springfield. Vırgınıa 22161 


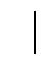


A magnesium alloy has been quasi-statically cycled elastically between zero load and tension. Both loading and unloading stress delays are found, and the unloading stress delay is further studied. An analytical expression is written for the unloading stress delay which is an elastic constitutive parameter. The potential use of these results for the acoustic emission monitoring of elastic stress states is discussed. 
INTRODUCTION

Acoustic emission (AE) is the generation, propagation and detection of transient elastic strain waves in materials as they undergo deformation or fracture. During the pust few years, AE has emerged as a testing method for materials research and as a monitoring technique for structural integrity assessment [1]. In 197.3 we suggested that for some materials the $\mathrm{AE}$ behavior is characteristic of the elastic stress state of the material, and that by perturbing that stress state, the resulting $\mathrm{AE}$ could provide information about the stress state [2]. This paper represents one of the important requirements of that concept as we present data and analytical expressions of AE/elastic stress constitutive relations which could be used toward the goal of the AE monitoring of structures which are elastically loaded.

$\mathrm{AE}$ may be generated during both loading and unloading. $\mathrm{AE}$ during loading has received by far the greater research attention. Associated with loading $\mathrm{AE}$ is the so-called "Kaiser effect" which is the irreversibility of $\mathrm{AE}$ from a body that has been stressed in tension, such that any subsequent loading results in $A E$ only if the previous maximum stress is exceeded [3]. The much less studied unloading $A E$ was mentioned briefly by Schofield [4], and further investigated by Kerawalla [5], Mitche11 [6], Agarwal [7], and Sankar [8]. Recently, 
both loading and unloading $A E$ from a magnesium alloy were studied by Williams and Emerson [9] during a program that was concerned exclusively with macroscopically elastic behavior. In that paper the existence of $\Lambda E / e l a s t i c$ stress constitutive relations was confirmed. (Also, it was noted that magnesium does not observe the Kaiser effect). This paper represents an extension of that research and it provides an important step toward the elastic stress monitoring described in [2]. 
The loading apparatus consisted of a hydraulic tensile loading device which produced a prescribed loading pattern and which was controlled by an electronic feedback system. The $\mathrm{AE}$ monitoring equipment was produced by Acoustic Emission Technology Corp. A PZT sensor (AC175) having a resonant frequency of $175 \mathrm{kHz}$ was used. The AE signal was band-pass filtered between 125 and $250 \mathrm{kHz}$. The total system gain was $100 \mathrm{db}$ and a fixed threshold voltage of $0.6 \mathrm{~V}$ after amplification was maintained. The minimum detectable stress pulse for this system has been calculated to be about $1.9 \times 10^{-9} \mathrm{MN} / \mathrm{m}^{2}$.

The specimen was made of AZ31B-F wrought magnesium alloy in a circular cylindrical form having a gage length of $7.62 \times 10^{-2} \mathrm{~m}$ and a gage section diameter of $7.62 \times 10^{-3} \mathrm{~m}$. The specimen was isolated from the loading grips with nylon pads and the sensor which was located at one end face of the specimen was held in contact by a force of $22 \mathrm{~N}$ which was exerted by a coil spring. A viscous acoustic couplant (AET SC-6) was used at the sensor-specimen interface. The specimen which was heat treated at $260^{\circ} \mathrm{C}$ for $1.2 \times 10^{3} \mathrm{sec}$ and air cooled, had a $0.2 \%$ offset tensile yield strength of $184 \mathrm{MN} / \mathrm{m}^{2}$.

* Reference [9] contains more details of the equipment and material. Thorough descriptions of the testing apparatus, including system parameters and calibration details, and the test material are given in $[10]$. 


\section{EXPERTMENTAL RESULTS}

The loading pattern consisted of loading the specimen at a rate of $186 \mathrm{MN} / \mathrm{m}^{2} / \mathrm{sec}\left(\dot{\sigma}_{L}\right)$, holding it at the prescribed upper stress level for $20 \mathrm{sec}$, and then unloading it completely at a rate of $476 \mathrm{mN} / \mathrm{m}^{2} / \mathrm{sec}\left(\sigma_{U}\right)$. This loading-unloading cycle was repeated to a total of 10 cycles with a $30 \mathrm{sec}$ interval at zero stress between each cycle.

A generic cumulative $\mathrm{AE}$ count result obtained during a loading-unloading cycle is shown in Fig. 1. Both loading AE and unloading $A E$ are denoted. Note that for both loading and unloading a certain amount of change of stress is required before $\mathrm{AE}$ is observed. These stress increments are defined as loading stress delay $\left(\sigma_{\mathrm{DL}}\right)$ and unloading stress delay $\left(\sigma_{\mathrm{DU}}\right)[5,9]$. Because metals are generally thought to behave elastically during unloading, it is this aspect of $\mathrm{AE}$ which we find particularly intriguing and which we shall examine here.

Fig. 2 is a typical plot of the cumulative unloading AE and the unloading stress delay for each of the 10 loading cycles. It can be observed that the cumulative unloading $A E$ varies consid-erably while the unloading stress delay is relatively constant. Thus, the stress delay appears to be a cycle-independent parameter, so it is chosen for emphasis in this presentation*.

* Note that this statement does not imply that the cumulative unloading $A E$ can not (or should not) be considered as a material constitutive parameter. Also, as shown in [9], the cumulative unloading $\mathrm{AE}$ approaches a constant value at a greater number $(\sim 30)$ of cycles. 
Fig. 3 gives the unloading stress delay results for a range of elastic loading-unloading sequences. The specimen was loaded to the stress corresponding to point $a_{1}$ for 10 cycles and the average unloading stress delay was recorded. The upper stress level was increased corresponding to point $a_{2}$ and the average unloading stress delay corresponding to $\mathrm{a}_{2}$ was observed. The upper stress level was increased again, and subsequently the data represented by the solid circles were generated and the straight line A was fitted. The specimen was then left completely unloaded for $24 \mathrm{hr}$. Then, the specimen was loaded to point $b_{1}$ and the average unloading stress delay for 10 cycles was recorded. The upper stress level was decreased to $b_{2}$ and the average unloading stress delay for 10 cycles was recorded. The upper stress level was decreased again, and subsequently the data represented by the crosses were generated and the straight line $B$ was fitted. The specimen was then left completely unloaded for $24 \mathrm{hr}$. Then, the specimen was loaded to point $c_{1}$ and the average unloading stress delay was recorded. The upper stress level was decreased to $c_{2}$, and the data represented by the triangles were generated and the straight line $\mathrm{C}$ was fitted. The specimen was left completely unloaded for $24 \mathrm{hr}$. The specimen was then loaded to point $d_{1}$ and the corresponding data were obtained as in the cases of lines $B$ and $C$.

In fitting straight lines to the data in Fig. 3, the method of least squares was used. The resulting expressions for the unloading stress delay are given in Table 1. 


\section{TABLE 1}

LXPRESSIONS FOR THE UNLOADING STRESS DELAY

GENERATED BY THE METHOD OF LEAST SQUARES

(Units: $\mathrm{MN} / \mathrm{m}^{2}$ )

$$
\begin{array}{ll}
\text { A-Data: } & \sigma_{\text {DU }}=0.0321 \sigma+29.67 \\
\text { B-Data: } & \sigma_{D U}=0.1897 \sigma+6.90 \\
\text { C-Data: } & \sigma_{D U}=0.1894 \sigma+11.80 \\
\text { D-Data: } & \sigma_{D U}=0.2035 \sigma+17.02
\end{array}
$$




\section{DISCUSSION}

It is observed that in Fig. 3, points $b_{1}, c_{1}$ and $d_{1}$ correspond very closely to points along line A. Also, lines B, C and D have nearly the same slope. The lines A, B, C and D were all determined in exactly the same manner with a single exception, namely the value of maximum stress in the "recent"* history prior to unloading which is defined as $\sigma_{\max }$. The values of $\sigma_{\max }$ for curves $B, C$ and D correspond to points $b_{1}, c_{1}$ and $d_{1}$, respectively. On the other hand, because of the increasing loading sequence, every point along line A represents a value of $\sigma_{\max }$.

In accordance with the observations in the previous paragraph, it is assumed that the unloading stress delay $\sigma_{\mathrm{DU}}$ is a function of the recent maximum stress which is defined as $\sigma_{\max }{ }^{*}$ The lines $A, B, C$ and D in Fig. 3 are reproduced schematically as solid lines in Fig. 4. Defining slopes $S_{1}$ and $S_{2}$ as indicated where lines $B, C$ and $D$ have the same slope $S_{1}$, the solid lines $A, B, C$ and $D$ can be expressed as

$$
\sigma_{\mathrm{DU}}=\mathrm{S}_{1} \sigma+\sigma_{\mathrm{DU}}^{\circ}-\left(\mathrm{S}_{1}-\mathrm{S}_{2}\right) \sigma_{\max }
$$

* For the purposes intended here, "recent" is defined as time periods which are much less than $10^{5} \mathrm{sec}$. This definition of "recent" is a material parameter for the magnesium alloy used in these experiments. 
where $\sigma_{\mathrm{DU}}^{\circ}$ is the unloading stress delay intercept of line $A$ and where $\sigma$ is the upper stress level for any current unloading cycle. Note that for line A where $\sigma_{\max }$ is equal to $\sigma$, equation (1) simplifies to

$$
\sigma_{\mathrm{DU}}=\sigma_{\mathrm{DU}}^{0}+\mathrm{S}_{2} \sigma
$$

The quantities $S_{1}, S_{2}$ and $\sigma_{D U}^{\circ}$ are material parameters which also depend upon loading variables such as unloading rate and holding times at both $\sigma$ and zero stress. Furthermore, these parameters as well as others discussed here may depend upon the AE monitoring system itself. Thus, as already indicated in [9] and [11] there is an important need for the development of standards for AE monitoring systems, testing procedures, and technical reporting. Equation (1) can be represented graphically by introducing a hypothetical line $E$ of slope $S_{1}$ which intersects the ordinate at $\sigma_{\mathrm{DU}}^{\circ}$ as shown in Fig. 4*. In order to obtain the unloading stress delay at a stress level $\sigma_{1}$, vertical lines through $\sigma_{1}$ and $\sigma_{2}$ are drawn where $\sigma_{2}$ is $\sigma_{\max }$ which for convenicnce is assumed to be known. The value of $\sigma_{D U}$ corresponding to $e_{1}$ represents the first two terms on the right-hand side of equation (1) and the value of $\sigma_{\mathrm{DU}}$ corresponding to $\mathrm{e}_{2}$ represents the third term in equation (1). So, by subtracting $e_{2}$ from $e_{1}$, the unloading stress delay may be obtained.

* Line $\mathrm{E}$ is hypothetical because it represents $\sigma_{\mathrm{DU}}$ for $\sigma_{\max }$ equal to zero which, of course, is not meaningful in the context described here. 
Assuming that the material in a given structure has been characterized with respect to the parameters $S_{1}, S_{2}$ and $\sigma_{D U}^{\circ}$, equations (1) and (2) represent relations which will likely be useful for determining residual or statically loaded stresses where a proposed trigger/AE monitoring system would perturb the stress state and observe the resulting $A E$ characteristics [2]. In the simplest case, the trigger may be uscd to produce an unloading of tensile stress and the corresponding unloading stress delay $\sigma_{D U}$ could be measured. If $\sigma_{\max }$ were known, the tensile stress prior to unloading could be determined directly from equation (1). On the other hand, equation (1) may be used to find $\sigma_{\max }$ if $\sigma$ is known. A test such as this could prove useful in cases where an accidental overload is suspected. Finally, because the parameters $S_{1}, S_{2}, \sigma_{D U}^{\circ}$ and thus $\sigma_{D U}$ itself may depend upon the $\mathrm{AE}$ monitoring system, they are not material constitutive parameters in the strict sense. Until any contributions of both the loading apparatus and monitoring system are removed from the reported values, they should more appropriately be defined as "pseudo-constitutive" parameters. Thus, the proper AE characterization of materials for the purposes discussed here as well as many others must await the development of more versatile $\mathrm{AE}$ equipment and standards for $\mathrm{AE}$ testing and reporting. 
CONCLUSIONS

A magnesium alloy has been loaded elastically in tension and then unloaded. It was found that a fixed amount of stress increment is required before $\mathrm{AE}$ activity is detected. This stress increment for increasing stress is called the loading stress delay and for decreasing stress, the unloading stress delay. As observed in [9], it is noted here also that magnesium does not observe the "Kaiser effect".

It has been shown that the unloading stress delay is an elastic constitutive parameter that is cycle-independent. The unloading stress delay depends on the upper stress level, any higher stress level undergone in the recent history of the material, and the loading pattern. It has been discovered that for a fixed loading pattern, a relatively simple analytical expression can be fitted to the unloading stress delay data.

A discussion of the applicability of these results including the analytical expression for the purpose of stress level evaluations has been given. Thus, with a trigger that can generate a stress increment slightly greater than the stress delay and with adequate $A E$ monitoring equipment, residual stress, static operating stress levels, and recent stress overloads can be determined.

A magnesium alloy has been used for these experiments because (1) compared with other metals magnesium generates a large amount 
of $\mathrm{AE}$ activity and (2) magnesium is predicted to become one of the major structural materials of the future [12]. Because unloading AE has been observed from other metals also $[4-8,10]$, the results reported here appear to be conceptually applicable to a range of materials. 
REFERENCES

1. R.G. Liptai, D.O. Harris and C.A. Tatro, "An Introduction to Acoustic Emission", Acoustic Emission, ASTM STP 505, American Society for Testing and Materials, 1972, pp. 3-10.

2. J.H. Williams, Jr., and S.S. Lee, "Monitoring of Elastic Stresses by Acoustic Emission", International Journal of Nondestructive Testing, 1977.

3. J. Kaiser, "Untersuchungen uber das Auftreten von Gerauschen beim Zugversuch", Dr.-Ing. Thesis, Technische Hochschule, Munich, 1950.

4. B.H. Schofield, "Acoustic Emission Under Applied Stress", Technical Documentary Report No. ASD-TDR-63-509, Part II, Wright Patterson Air Force Base, May 1964.

5. J.N. Kerawalla, "An Investigation of the Acoustic Emission from Commercial Ferrous Materials Subjected to Cyclic Tensile Loading", $\mathrm{Ph} . \mathrm{D}$. Thesis, University of Michigan, 1965.

6. L.D. Mitche11, "An Investigation of the Correlation of the Acoustic Emission Phenomenon with the Scatter in Fatigue Data", Ph.D. Thesis, University of Michigan, 1965.

7. A.B.L. Agarwa1, "An Investigation of the Behavior of the Acoustic Emission from Metals and a Proposed Mechanism for its Generation", Ph.D. Thesis, University of Michigan, 1968.

8. N.G. Sankar, "Unload Emission Behavior of Materials and its Relation to the Bauschinger Effect", Ph.D. Thesis, University of Michigan, 1969.

9. J.H. Williams, Jr., and G.P. Emerson, "Constitutive Acoustic-Emission Elastic-Stress Behavior of Magnesium Alloy", NASA CR-2839, 1977.

10. G.P. Emerson and J.H. Williams, Jr., "Acoustic Emission from Elastically Stressed Metals", Composite Materials and Nondestuctive Evaluation Laboratory, Department of Mechanical Engineering, Massachusetts Institute of Technology, August 1976.

11. J.H. Williams, Jr. and S.S. Lee, "Acoustic Emission Monitoring of Fiber Composite Materials and Structures", Composite Materials and Nondestructive Evaluation Laboratory, Department of Mechanical Engineering, Massachusetts Institute of Technology, January 1977.

12. "Trends in Usage of Magnesium", National Materials Advisory Board (NAS-NAE), NTIS No PB-254 189. 


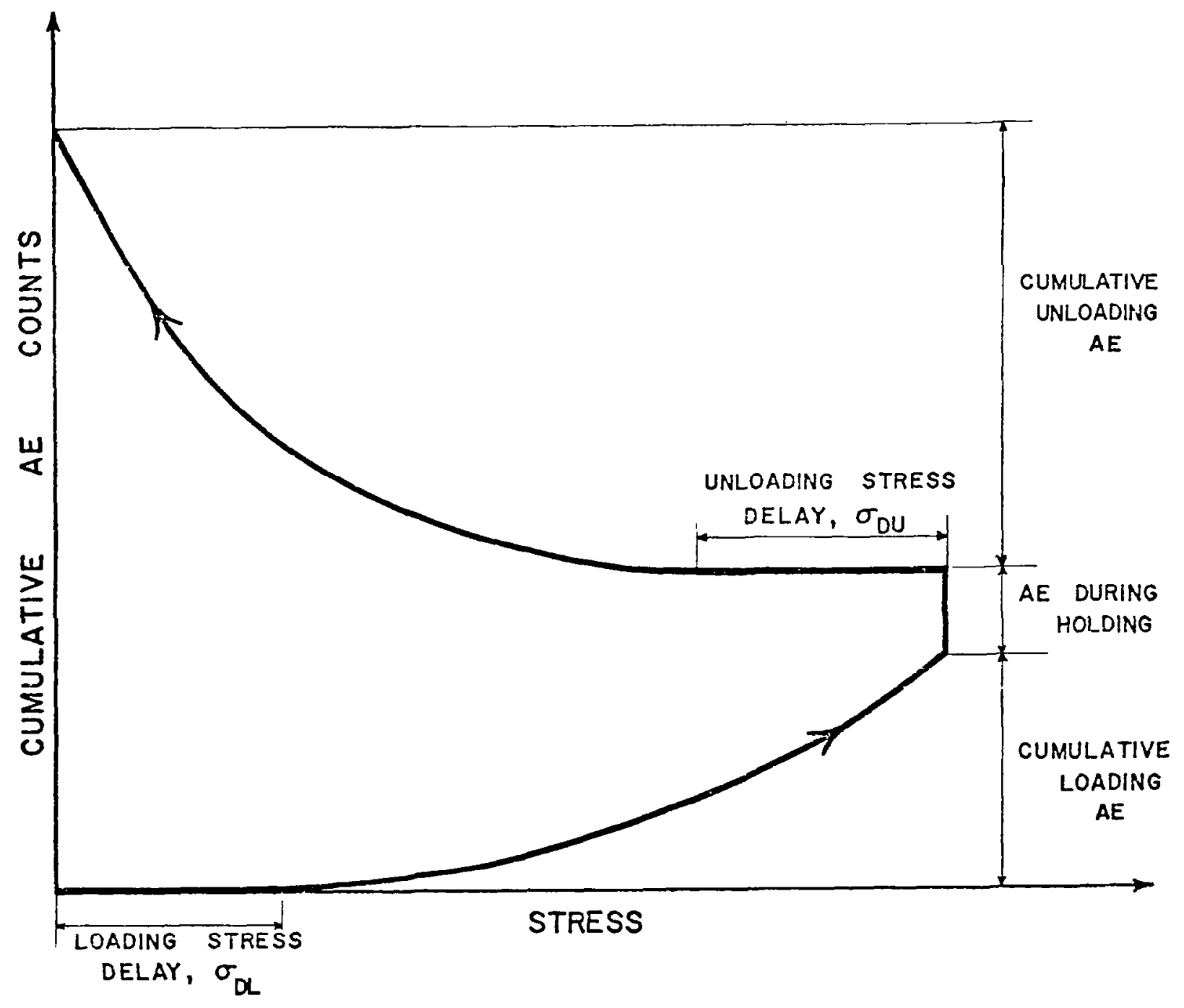

Fig. 1 Generic cumulative AE counts for a loading-unloading cycle. 


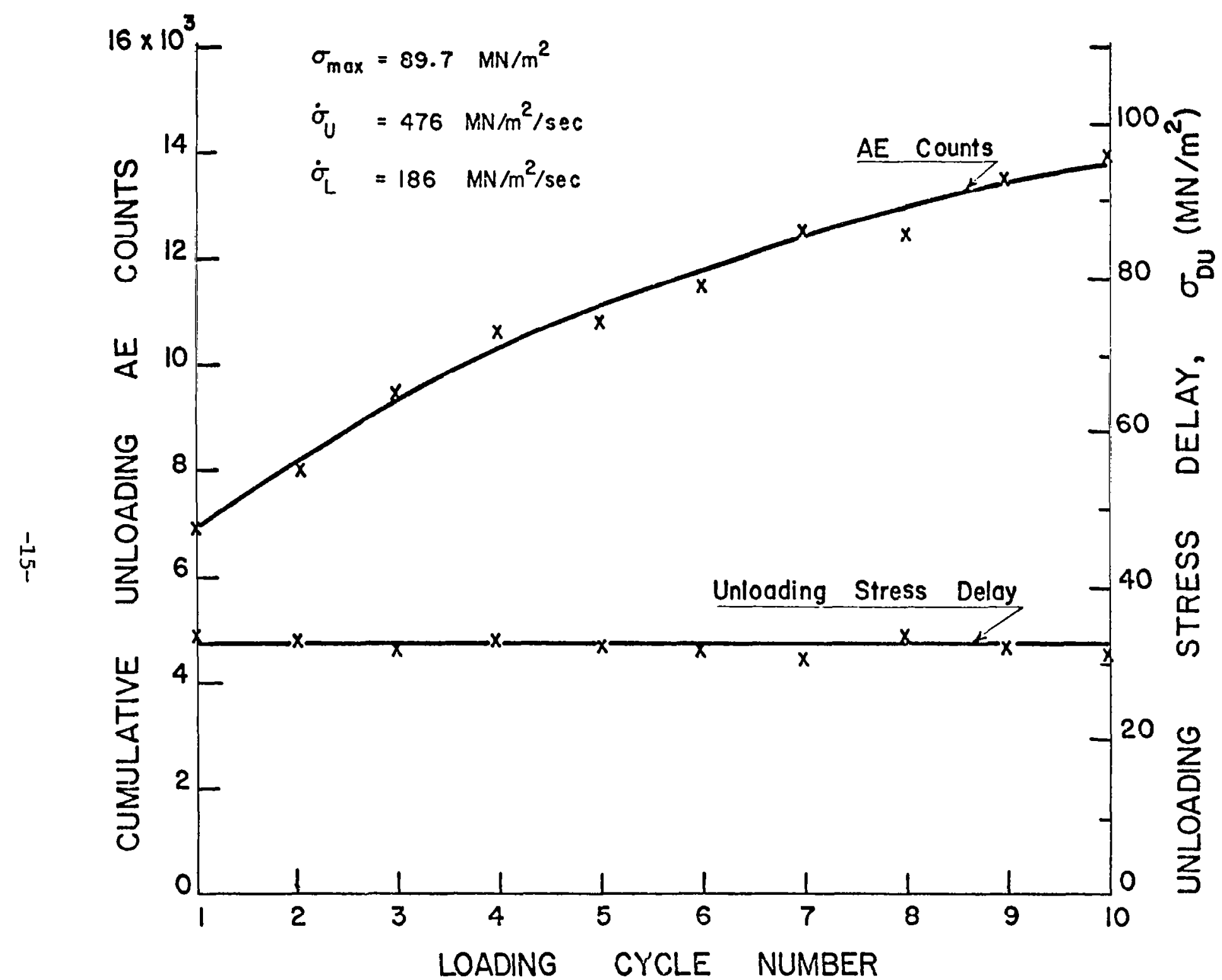

Fig. 2 Cumulative $A E$ counts and unloading stress delay as a function of sequential loading cycles. 


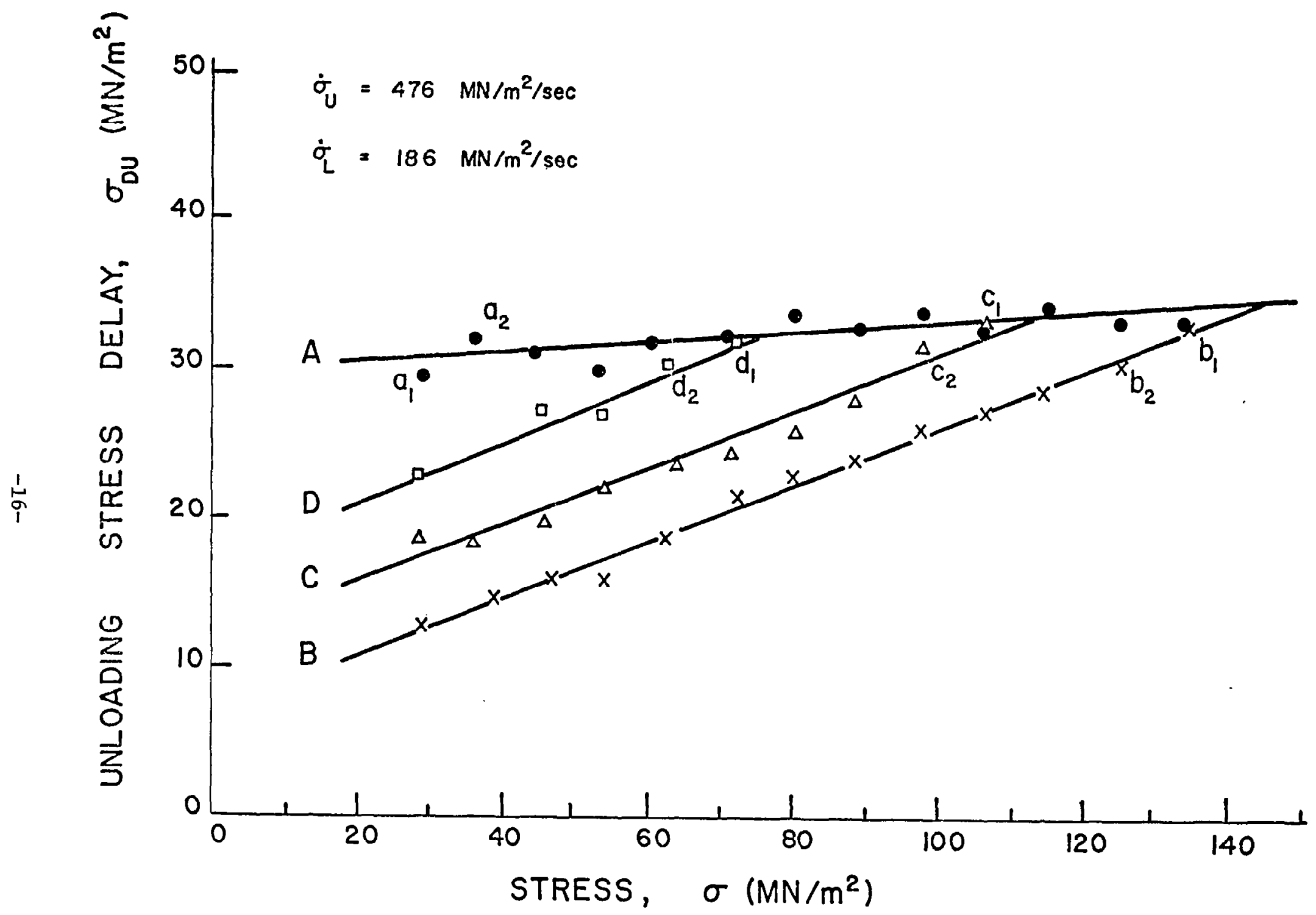

Fig. 3 Average unloading stress delay for increasing upper stress levels (Curve A) and decreasing upper stress levels (Curves B, C and D). 


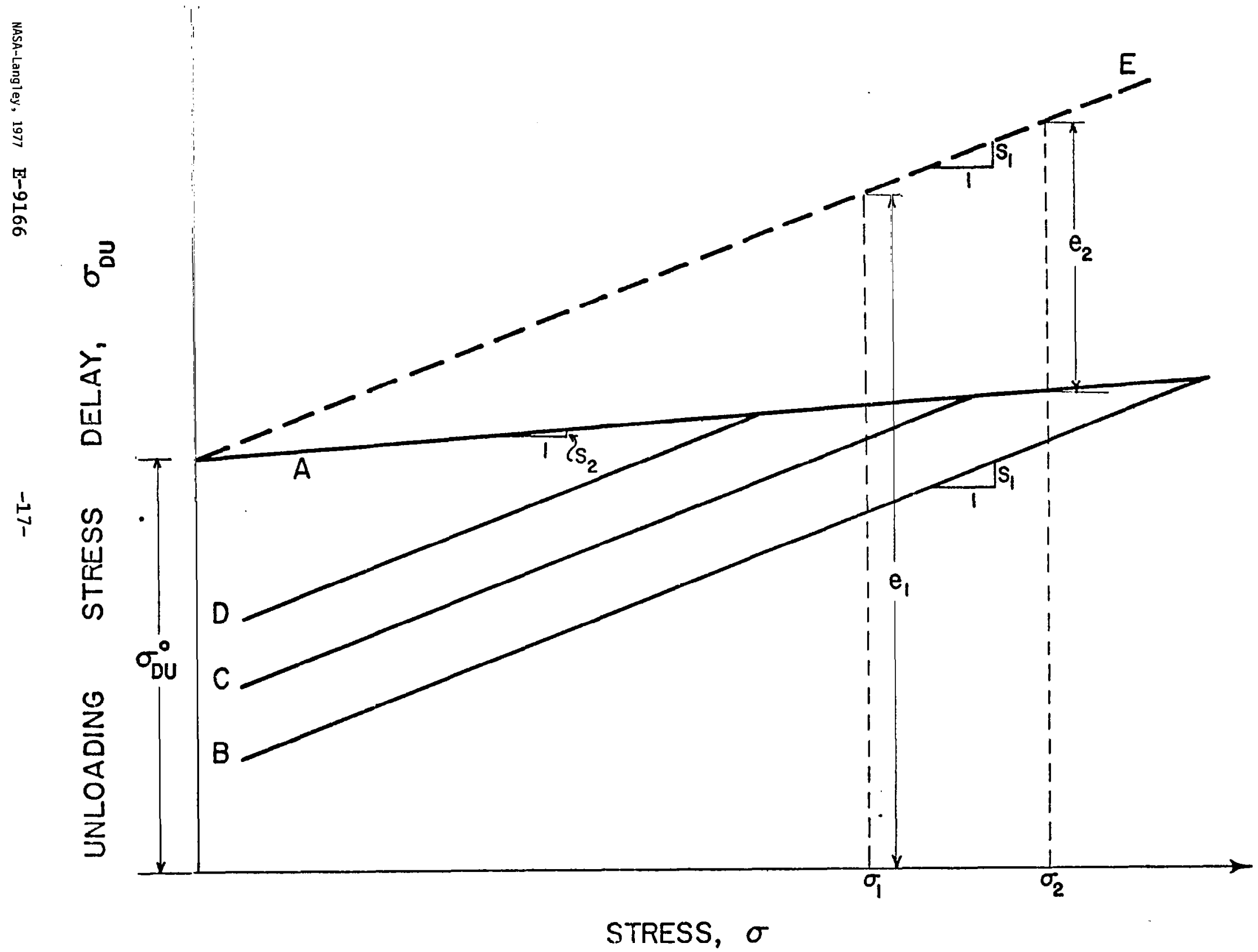

Fig. 4 Schematic illustrating parameters relating unloading stress delay and stress levels. 